\title{
Correction to: Comparative analysis of COPD associated with tobacco smoking, biomass smoke exposure or both
}

Jordi Olloquequi ${ }^{*}$, Sergio Jaime ${ }^{2}$, Viviana Parra ${ }^{2}$, Elizabeth Cornejo-Córdova ${ }^{3}$, Gonzalo Valdivia ${ }^{4}$, Àlvar Agustí $^{5}$ and Rafael O. Silva ${ }^{2}$

\section{Correction}

In the original publication [1] there is an error in the FEV1/ FVC values found in Table 1 . The correct version can be found in this Erratum.

\begin{abstract}
Author details
'Instituto de Ciencias Biomédicas, Facultad de Ciencias de la Salud, Universidad Autónoma de Chile, 5 Poniente \#1670, 3460000 Talca, Chile. ${ }^{2}$ Unidad Respiratorio, Centro de Diagnóstico Terapéutico, Hospital Regional de Talca, 1 Norte \#1990, 3460000 Talca, Chile. ${ }^{3}$ Subdepartamento de Salud Laboral, Hospital Regional de Talca, 1 Norte \#1990, 3460000 Talca, Chile. ${ }^{4}$ Departamento de Salud Pública, Facultad de Medicina, Pontificia Universidad Católica de Chile, Av. Libertador Bernardo O'Higgins \#340, 35420000 Santiago, Chile. ${ }^{5}$ Respiratory Institute, Hospital Clínic, Institut d'Investigacions Biomédiques August Pi i Sunyer (IDIBAPS) Universitat de Barcelona, Rosselló \#149-153, 08036 Barcelona, Catalonia, Spain.
\end{abstract}

Published online: 30 April 2018

\section{Reference}

1. Comparative analysis of COPD associated with tobacco smoking, biomass smoke exposure or both. Respir Res. 2018;19:13. https://doi.org/10.1186/ s12931-018-0718-y

\footnotetext{
*Correspondence: jordiog82@gmail.com

${ }^{1}$ Instituto de Ciencias Biomédicas, Facultad de Ciencias de la Salud,

Universidad Autónoma de Chile, 5 Poniente \#1670, 3460000 Talca, Chile

Full list of author information is available at the end of the article
} 
Table 1 Demographic and clinical data

\begin{tabular}{|c|c|c|c|c|}
\hline & $\begin{array}{l}\text { Control Subjects } \\
n=52\end{array}$ & $\begin{array}{l}\text { TS COPD } \\
n=49\end{array}$ & $\begin{array}{l}\text { BS COPD } \\
n=31\end{array}$ & $\begin{array}{l}\text { TS + BS COPD } \\
n=46\end{array}$ \\
\hline Sex, Male (\%)/Female (\%) & $15(29) / 37(71)$ & $35(71) / 14(29)$ & $12(39) / 19(61)$ & $26(63) / 20(37)$ \\
\hline Age, years & $70.34 \pm 5.95$ & $69.41 \pm 8.69$ & $72.29 \pm 9.49$ & $69.93 \pm 7.19$ \\
\hline Smoking history, pack-years & - & $41.57 \pm 25.62$ & - & $55.46 \pm 47.12$ \\
\hline Biomass exposure, hour-years & - & - & $340.90 \pm 206.09$ & $345.15 \pm 193.16$ \\
\hline Scolarship, years & $13.21 \pm 2.06$ & $7.56 \pm 4.25^{\mathrm{a}}$ & $5.20 \pm 3.59^{a, b}$ & $6.09 \pm 3.93^{a, b}$ \\
\hline $\mathrm{BMI}, \mathrm{kg} / \mathrm{m}^{2}$ & $30.09 \pm 5.54$ & $27.67 \pm 5.08^{a}$ & $26.57 \pm 3.06^{\mathrm{a}}$ & $27.35 \pm 5.69^{a}$ \\
\hline Exacerbations in the previous year & - & $1.10 \pm 1.37$ & $0.58 \pm 0.42$ & $0.69 \pm 1.29$ \\
\hline $\mathrm{FEV}_{1}, \%$ predicted & $110.19 \pm 17.46$ & $56.88 \pm 19.37^{a, c}$ & $68.09 \pm 32.30^{a}$ & $53.79 \pm 18.67^{a, c}$ \\
\hline $\mathrm{FEV}_{1} / \mathrm{FVC}, \%$ & $84.46 \pm 4.24$ & $54.25 \pm 11.17^{a}$ & $58.39 \pm 8.62^{a}$ & $53.53 \pm 12.35^{a, c}$ \\
\hline $\mathrm{DL}_{\mathrm{CO}}, \%$ predicted & $81.52 \pm 21.21$ & $66.60 \pm 19.82^{a}$ & $73.73 \pm 17.16$ & $61.22 \pm 24.98^{a, c}$ \\
\hline Oxygen Saturation, \% & $96.91 \pm 1.36$ & $92.65 \pm 4.55^{\mathrm{a}}$ & $93.94 \pm 4.04^{\mathrm{a}}$ & $90.52 \pm 4.90^{a, b}, c$ \\
\hline $6 \mathrm{MW}$, meters & $492.48 \pm 78.51$ & $355.96 \pm 163.02^{\mathrm{a}}$ & $375.29 \pm 143.75^{a}$ & $344.09 \pm 161.12^{\mathrm{a}}$ \\
\hline $\mathrm{mMRC}$ & - & $2.38 \pm 1.47$ & $2.37 \pm 1.16$ & $2.67 \pm 1.03$ \\
\hline CAT & - & $15.49 \pm 8.21$ & $14.84 \pm 6.69$ & $13.18 \pm 6.69$ \\
\hline BODE & - & $2.88 \pm 5.71$ & $2.71 \pm 6.35$ & $3.87 \pm 7.72$ \\
\hline
\end{tabular}

Data presented as mean \pm standard deviation, unless otherwise indicated. Definition of abbreviations: $B M I$ body-mass index, FEV forced expiratory volume in $1 \mathrm{~s}$, FVC forced vital capacity, $D L_{C O}$ carbon monoxide diffusing capacity, 6 MW 6 min walking test, $m M R C$ modified Medical Research Council scale, CAT COPD assessment test, $B O D E$ Body-mass, airflow Obstruction, Dyspnea and Exercise index

${ }^{\mathrm{a} D i f f e r e n t}$ from control subjects $(p<0.05$, by ANOVA)

${ }^{\mathrm{b} D i f f e r e n t ~ f r o m ~ T S ~ C O P D ~}(p<0.05$, by ANOVA)

'Different from BS COPD $(p<0.05$, by ANOVA $)$ 